\title{
Based on the digital technology of smoked painting cultural creation product design
}

\author{
ZhangJing $^{1}$ a YinJiayi $^{2}$ b* $^{*}$ \\ ${ }^{1}$ School of Art and Design Xi'an University of Technology Xi' an, China \\ ${ }^{2}$ School of Art and Design Xi'an University of Technology Xi' an, China
}

\begin{abstract}
Under the product design in the digital information age is no longer a common product design, digital technology has a wide range of areas, is composed of a variety of technology, by referencing the digital technology in the design of the product to solve the current existing problems and solutions. Through the understanding of the current situation, color analysis, image extraction of these three artistic processing design this design contains more designers to understand the deep step of our era, the design focuses on modern people psychological needs and the moral of the product. Gradually integrate product design art and digital technology into people's life, making it a key factor to promote the development of digital art technology.
\end{abstract}

\section{Smoked painting current situation and market analysis}

\subsection{Current situation}

With the development of society and the rise of industrial civilization, the influence and impact of industrial civilization on the traditional Chinese cultural industry has led to the loss of folk smoked painting art. The proportion of folk art in people's life is becoming less and less, leading to the gradual neglect of the existence of smoked painting art by ordinary people. Cultural and creative products are an emerging industry with creativity as the core under the global cultural background. Therefore, unique cultural characteristics are integrated into the daily necessities of modern people to reflect the richness and culture of products. But for now, smoked painting type single, machine-made, adornment is the traditional form of drawing, smoked painting of wen gen product design and promotion is also less, basic implementation, cannot reflect the local characteristics and cultural connotation, also didn't match with the current young people's aesthetic consciousness, to a certain extent, affected the young people's purchase intention. At present, there are the following problems:

In the first place, innovation being not enough. At present, most of the smoked paintings are just copying, cloning, or borrowing the traditional paintings, simply through changing the surface composition and basic signs, without exploring the application of smoked paintings and local cultural traits from different aspects and product levels. Therefore, cultural and creative products are not enough in innovation, and it's not very well received by tourists.

In the second place, handicraft thinking outweighing

a756225884@qq.com

b1040197066@qq.com product design thinking. Without research and analysis on consumers' demands, smoked painting and cultural creation products are divorced from the real life demands, and its universal lifelike is not enough, their usability is not strong, and they lack practical purchase value. Therefore, the penetration and inheritance of product innovation are not enough. This is also one of the smoked painting reasons for the single type and poor quality of smoked painting art and cultural creation products.

In the last place, lack of systematic cultural products. There is deficency of substantial products related to cultural and creative products of smoked paintings, and no influential brand has been formed. The lack of regional folk culture features indicates that smoked painting's cultural exploration is not enough. The products are too single, the visual impact is not enough, and the distribution of product forms is not reasonable, which completely fails to arouse the interest of consumers.

\subsection{Market analysis of cultural and creative products of fumed paintings}

As a unique type of folk art in northern Shaanxi, smoked paintings contain abundant cultural connotations. In the context of cultural creativity, smoked painting culture can provide important cultural resources for tourism product design. At the same time, smoked painting as a cultural heritage, with special history and culture, forms a peculiar fond memory and nostalgia in the minds of many people. In addition, it is the carrier for traditional Chinese rural feelings. All over the country now great importance is attahced to the development of cultural and creative products, during which smoked painting 
development is just in the initial stage. This is a good opportunity for smoked paintings to get better development, hence the promotion of the development of tourism products.

\subsubsection{Market conditions}

Through field research, we found that smoked painting product market is almost a blank, although there is a local type of tourist souvenirs, but these souvenirs are not combined with smoked painting culture, most of the major is public goods, no uniformity, no local characteristics, its lack of cultural products with smoked painting art characteristics, it can not satisfy the consumer demand and spiritual needs for tourists.

\subsubsection{Analysis of consumer groups}

In recent years, the number of custom tourism in northern Shaanxi has increased. Through the investigation of the tourist group in 2019, the purchasing power of the group is analyzed to classify them according to gender, age, income, hobbies and other directions. Relatively speaking, men have more objective requirements for products, pursue the use value and simplicity of products, and have no clear requirements for price requirements, and do not have too much demand for the appearance of products and shapes. For women, it is often emotional consumption, they attention to ornamental value, choice of appearance. Therefore, smoked painting, in the product development design, should give full consideration to the tourists as well as the main body, the various requirements of consumers as possible to meet, on the basis of consumer psychology, tourism consumption subject fully considering regionality, culture, innovation and other factors, realize the cultural effects of cultural and creative product design and an organic unity of economic benefit.

Above all, smoked painting cultural and creative products have a certain market demand, to express in the design of regional culture and the connotation, combining product practical function and smoked painting art perfect combination, passed to the user through the impact of the visual effect, achieve the user's emotional needs and experience and national emotional resonance, in order to enhance the smoked cultural and creative products targeted, attract consumers' interest and appeal.

\section{Smoked painting art features}

Smoked painting appeared around the Qing Dynasty, especially coming from the smoked pattern technology in the paper-cutting. The smoked painting of Fu County in northern Shaanxi originates from the smoked sample of paper-cut, which is a twin-sister folk art of paper-cut and a decorative art. It is the decoration for the working people in northern Shaanxi in order to prevent mosquitoes and decorate the interior space. First of all is to use paper cut out different implicated pattern, eliminate evil, hope can to ward off bad luck will paper-cut is flat out on the white paper or color paper, rinse with water mist spraying is soaked, and then repeated with kerosene or birch bark smoked on hemp paper wet paper cutting, remove the paper-cut smoked to black, in the shelter dry in the shade, then lightly smoked off, reveal a black white picture, lively and simple but elegant pattern presented itself. Every festival occasion or to decorate a bedroom, a local farm women, the paper-cut, smoked, color painting, in the kang, bowl rack with colorful smoked painting, colourful, colour is gorgeous, manifests the lively atmosphere in festival occasion booming, already meet the demand of its own life, and reflects the masses of working people love of life on the northern shaanxi plateau, expressed a folk life, ideal performance.

\subsection{Modeling of Smoked paintings}

The smoked painting is concise in modeling, full and ample in composition, with rich story-based content. It takes the narrative expression and symbolic expression, formed the stylization and regular pattern, leaving a large area of blank, and added with lace, cured after leaving large pieces of black, between the form and form strong decoration. The most common form of decoration is moire continuous, each layer of smoked patterns are used to subflower or chain smoked painting, more to double number repeated arrangement, highlighting the beauty of balance, two pillars stick paper-cut applique, not only reflects the beauty of symmetry and harmony at the same time to increase the meaning of good luck.

\subsection{Traditional patterns of smoked paintings}

The patterns of smoked paintings are mostly symbolic and auspicious. For example, magpies for good news, rooster crowing at dawn, auspicious clouds, lotus, pines, mandarin ducks, carp, lotus, little chubby boys, etc., through which people can pray for happiness and health, thus reflects people's wish for happiness and a better life. Existing smoked paintings are improved through the ages, getting rid of the original witchcraft, totem worship, with only the good wish left for procuring blessings and evading disasters. Besides, there are some smoked paintings close to life, full of interest, shaping the artistic images beloved by the working people, or with widely spread historical figures and wonderfully moving legends, opera characters and popular love stories, and so on. (table 1) 
TABLEI. Fumigation Painting Pattern Elements (made by the author)

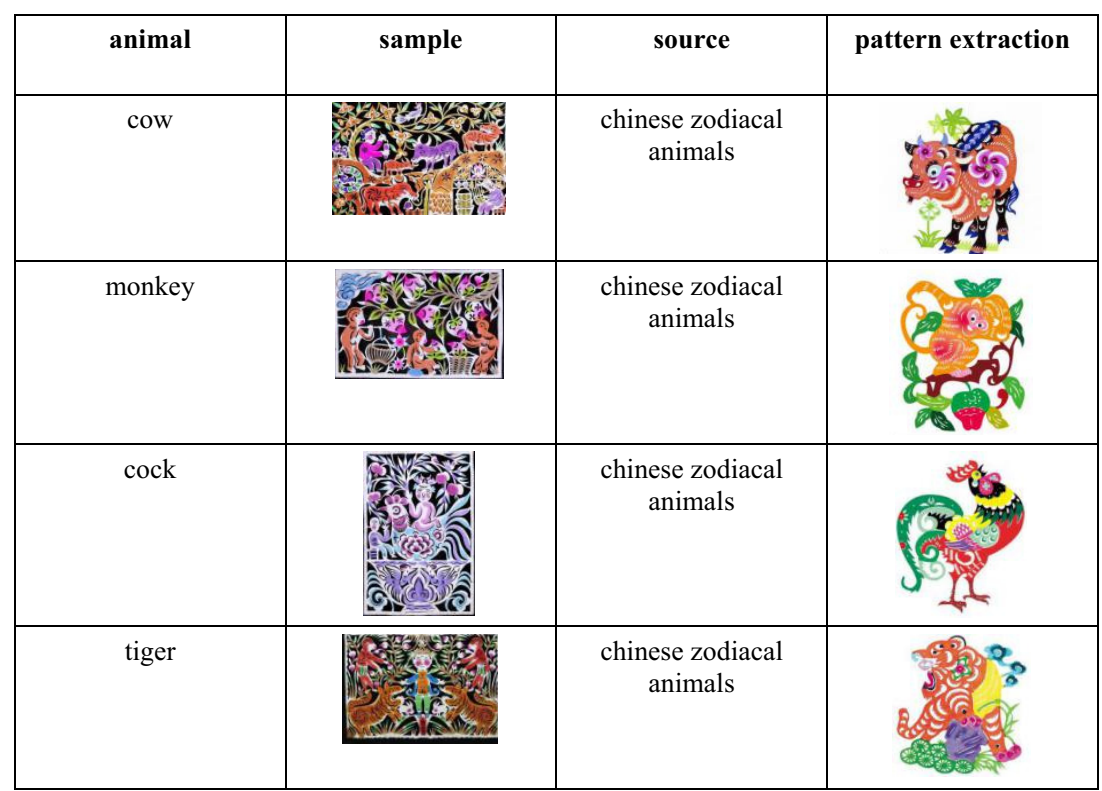

\subsection{Color choosing of smoked paintings}

Smoked painting is made by curing it with fire, with black and white the fundamental colors. And colors are congruous and soft, like black flannelette. The contrast in colors of smoked painting is strong and bright. It uses the traditional five colors and has symbolic characteristics. It is caused by the combination of people's color psychological feeling and the color of smoked painting. Early, smoked painting using only black and white colors, then with the development and progress of the society, a large number of colors such as light yellow, earthy yellow, perhaps because of the loess plateau nourish $\mathrm{Fu}$ county ancestors of the people, so will be a lot of application in each smoked painting to yellow, the women seem to prefer this color. But it is still dominated by black and white. Embellish blue, green, it hope to add a touch of embellishment for plain life with the color of life vitality. The meaning symbolized by red is intertwined with the will of the people. Also conform to the northern shaanxi folk wisdom of "soft on hard, color not stare blankly" color formula, according to the folk culture red and yellow represent happy events, defined as hard colo. Yellow, green and pink is defined as a soft color. match Pair the hard color with the soft color, make the picture presents colorful, gives people a strong visual impact of the profound meaning of effect, with a jubilant sense.

\section{The cultural and creative design of fumed paintings}

\section{1 motion-based design concept}

In order to impress consumers, the most important factor for cultural and creative products is to integrate emotions that can resonate with consumers, so that cultural and creative products can build a bond of emotional communication with consumers, arouse their emotions, and enhance their purchase intention and enthusiasm in this way. Therefore, starting from the characteristics of smoked painting modeling, the extraction of modeling features, gradually evolved into a stronger sense of decoration, make the decoration and traditional culture and daily necessities combined. Make people of all ages and different levels of education can accept and like the cultural and creative products. The folk art forms with characteristics of the Loess Plateau in northern Shaanxi can arouse consumers' cultural identity, which maximizes consumers' inner feelings through such design concept, and increases the collection value of cultural and creative products to a certain extent. At the same time, it also strengthens the regional recognition of products, promotes the confidence of regional culture, and creates a strong emotional resonance between cultural and creative products and consumers both emotionally and visually.

\subsection{Design principles}

In the first place, the combination of practicality and aesthetics. The perfect combination of practical value and aesthetic value in the process of product design is the basic principle of cultural and creative product design. First by summing up all sorts of modelling of smoked painting symbol, for extraction, refining, restructuring and reengineering to form art elements that are in accordance with the time development, again, rearranging themselves through different ways to get new cultural elements, using some technology method to group a perfect fusion of product and smoked painting art characteristics of wen gen products shall be issued. It not only retains the original charm of its pattern, but also conveys cultural information in this way, which not only realizes the value of the product itself, but also shows the unique characteristics of culture. The patterns and patterns can be extracted from the works, and the techniques, techniques and artistic modeling can be extracted from the crafts. 
In the second place, the combination of region with culture. The way of making art elements and folk customs make the art of smoked painting have their own unique regional cultural characteristics and strong regional identification. Combining culture and fashion, not only can good intention to spread culture, also can extend the meaning of the products, improve the text and the product's economic value, cultural value and collection value, it can also enhance attraction of the product, while emphasizing the recognition of smoked painting, there is also a strong sense of art, this accords with the principle of combining recognition and artistry.

In the last place, combination of innovation with the times. In order to know the needs of different people, the creative design of smoked painting should follow closely with social and conform to the modern aesthetic point of view. Cartoon image and two continuous artistic treatment, combined with pattern elements as shading. It shows the concept of keeping pace with social and diversified styles, gives smoked painting elements innovative cultural details and novel design concepts, and spreads its own cultural concepts and characteristics to the society, so that the image of smoked painting not only has national charm but also conforms to the development of the social.

\subsection{Product creative design process}

The shape and cultural expression of smoked painting has pure and simple artistic characteristics, and its extensive and profound cultural deposits have attracted worldwide attention, which is a valuable resource of original ecological culture. Firstly, collect and sort out the classic patterns of smoked painting, extract the elements of the shapes and patterns of the familiar regional sense symbols, and redesign them according to the colors of smoked painting to obtain the decorative elements of smoked painting's cultural and creative products. For example, in the design, the form, color and auspicious meaning of smoked painting are mainly extracted. The background color is black, the pattern is exaggerated child modeling, and the color is yellow similar to that of the Loess Plateau, forming simple regional national characteristics, which is combined with the modern mobile phone case. It improves the quality and quality of smoked painting cultural and creative products, it can also brings user unique touch and visual experience.

\subsubsection{Decoration for daily necessities}

As a unique regional culture in northern Shaanxi, smoked painting in Fu county has a wide range of themes and carries abundant cultural connotations, providing important cultural resources for cultural and creative product design. Taking cultural and creative products as the entry point, it extends to the decoration of various products in daily life, which is conducive to the protection and inheritance of China's soon-to-be lost cultural heritage. For example, ornaments, articles of daily use, gifts, collection of high-quality goods, realize the characteristic development of daily necessities, combine traditional culture with modern daily necessities, and promote the circulation of smoked painting tourism cultural products.

In the design process of smoked painting's cultural and creative products, visual symbols of smoked painting are applied as decorative patterns to the development of daily necessities, such as cups, pillows and ornaments. This not only highlights the beauty of tourism derivatives, but also makes them conform to the characteristics of "interest", "native" and "emotional resonance". Abstract and summarize with modern ideas and modern expression forms, techniques, exaggerate, strengthen, decorate and reconstruct on this basis, which has achieved the purpose of design and transformed the traditional folk patterns into fashionable symbol elements. For example, the 12 zodiac elements in the creation of the smoked painting are not only endowed with good meanings, but also make the content of the smoked painting more lively. The patterns of 12 Chinese zodiacal animals in the smoked painting were summarized, extracted, and matched with the shape of the mobile phone shell. The patterns were printed on the mobile phone shell to gradually integrate into life and achieve the purpose of inheriting traditional culture and art.

At the same time it can also be applied in daily supplies, such as smoked painting pillow design. The 12 zodiac elements that are familiar to the public and the exaggeration technique are used to extract the blue and black collocation, while the red is a secondary color. So as to convey the good wishes of good luck. The color contrast that presents is intense, abound change, much and not miscellaneous, colourful and not common. Material selection of daily life fabric, easy to keep warm, soft and close-fitting, moisture absorption and good air permeability. It still can print on hoodie, short sleeve, canvas bag, it win the love of young people. It not only improves the practical value, but also innovates the cultural value. Such combination of design, on the one hand, makes the traditional arts and crafts appear in the life of modern people with a new attitude, and constantly influences people's understanding of traditional arts and crafts. On the other hand, it also drives the growth of local economy. (Fig. 1)
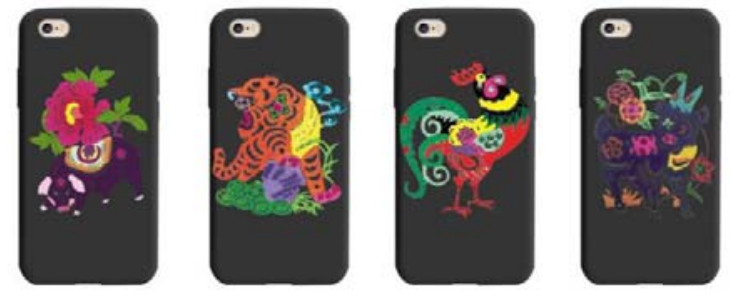

Figure 1. mobile phone shell design (made by the author)

\subsubsection{Integration of packaging design}

In packaging design, a large number of pictures are usually needed to reflect the product information or regional cultural connotation. In northern Shaanxi local specialty packaging, extracting smoked painting graphics, composition, again use the smoked painting regional 
color language, the moral will be given by graphic design, considering the influence of the emotional and psychological aspects, with a regional style product packaging series, form a certain aesthetic feeling of packaging, make these agricultural products not only enhance the brand class, but also increase the regional cultural connotation and fashion sense. (Fig. 2)
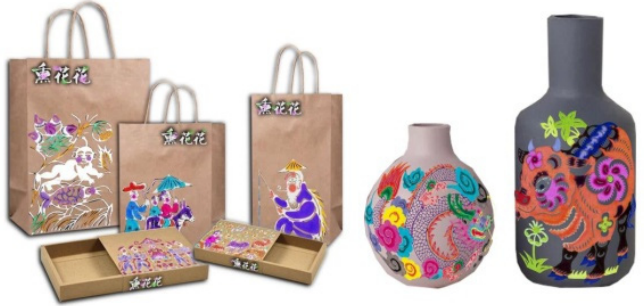

Figure 2. packaging design of gift box (made by the author)

\section{Conclusion}

Digital technology and wen gen product design is an emerging market, the market need more innovative design, with the improvement of people's living standard, people's demand for art products in quantity on artistic grade quality are continuously put forward new requirements of the new era, put the digital technology and smoked painting linked to the protection of cultural heritage, so that is conducive to the protection and inheritance the impending loss of cultural heritage, it also can make the traditional culture and art into our life, for the innovation of the smoked painting design brings the good impetus, it also brought a certain space for development, effectively improve the degree of product innovation and the development space of digital technology in cultural and creative products.

\section{ACKNOWLEDGMENT}

This research was financially supported by graduate school of Xi'an University of Technology, ShaanXi. Subject number: Practical Research on art Major Courses under the concept of "Three complete Education"

\section{References}

1. Highland, Tian Yilong. The influence and mission of One Belt And One Road initiative on the development of western art design and culture industry [J]. Design 2017 (23): 94-95.

2. Ning Yu, Rong Hua. Research on Shaanxi Folk Art [M]. Xi 'an: Shaanxi People's Fine Art Publishing House, 1988.

3. Xue Jiahui. Exploration of the Origin of Traditional Paper-cut fumigation Paintings on the Loess Plateau in the north of Weihe River [J]. Folk Art,2019:98-101.

4. Zhang Qiang. Analysis of Chinese Character Design Art from the Perspective of Deconstruction [J]. Collection, Writing and Editing,2017(05):63-64.

5. Chen Shaofeng. Deconstruction and Reorganization
-- A Ramble on Cultural And Creative Design Methods [J]. Research on Cultural Industry of Chinese Museums,2015(00):79-83.

6. Zhao Qin, Hui Xu. Application of Gantai Art Elements in Cultural and Creative Product Design [J]. Packaging Engineering. 2020(07)

7. Wang yanyan. Extraction and protection of local features of tourist souvenirs $[\mathrm{J}]$. Packaging engineering,2015,36(10): 148

8. Zheng Zhen. Innovative Application of Intangible Cultural Symbols in Tourist Souvenir Design -- A Case study of the Three Gorges Region of the Yangtze River [J]. Art Review, 2015 (10):83-86. 